\title{
una clasificación de suelos, basada en la granulometría, en relación con su aptitud para la mezcla con cemento
}

\author{
SANDRO ROCCI, Ingeniero de Caminos
}

$607 \cdot 3$

Uno de los más importantes factores que influyen en la dosiflcación minlma de cemento para obtener un suelo-cemento de caracteristicas dadas; $\mathrm{y}$ viceversa, en las propiedades de un suelocemento con una dosificación dada de cemento, es el tipo de suelo. Entre las características que definen este "tipo" de suelo, una primordial es la granuiometría, o distribución ponderal de los distintos tamaños.

Ha de recordarse que, cuanto más fino es un suelo, más aumenta su superficie especiflca (área de las partículas por unidad de peso), y que, por lo tanto, los fenómenos físico-químicos asociados con dicha superficie cobran mayor importancia. Siguiendo esta idea, podriamos distinguir el árido, inerte, grueso, del suelo, activo, fno. Naturalmente, la mezcla de estos dos típos opuestos de material, con cemento, no puede conducir a los mismos resultados.

Stempre en estos casos extremos, la adición de cemento en pequeñas dosis y siempre en las debidas condieiones de compactación y curado, produce dos efectos distintos:

- En los aridos (cuya prineipal característica es la de poseer rozamiento interno, aunque poca o ninguna cohesiónj el cemento une entre si a los granos, y sus vinculos forman una estructura celular que conflere cohesión al conjunto, aumentando, por lo tanto, su resistencia intrínseca.

- En los suelos propiamente dichos (que tienen el inconveniente de alterar su resistencia por la acción del agua) el cemento ejerce, ante todo, una intensa acción superficial que neutraliza en cierto modo la del agua, haclendo que el suelo asi "modiflcado" pierda su plastieidad, aumente su resistencia en estado húmedo, y pierda, en defnitiva, su susceptibilidad al agua.

Aumentando la dosificación de cemento se obtiene un nuevo material, cuyas propiedades no son ya tan sólo las de los áridos o suelos, modifacados o mejorados, sino que también presenta una nueva caracteristica que convierte a la mezcla en un material diferente: el suelo-cemento (en el caso de lós suelos) o el hormigón magro (en el caso de los áridos). Esta nueva propiedad es la durabilidad, o resistencia a la intemperie, a las alternancias de humedecimiento y desecación, $\mathrm{y}$ congelación y deshielo, que destruyen la cohesión entre las particulas $y$, por consiguiente, disminuyen grandemente la resistencia mécánica.

Naturalmente, el proceso que hemos descrito cualitativamente, no es cuantitativamente ldéntico para todos los suelos o áridos: de donde la necesidad de una clasificación.

Por lo pronto, hay materiales cuya misma naturaleza los bace no aptos para la mezcla con cemento, cualquiera que sea la dosiflcación de éste: entre los áridos, los que son excesivamente gruesos (elevado indice de huecos, compactación imposibie); entre los suelos, los excesivamente cohesivos (cuya mezcla con el cemento es imposible de realizarse homogéneamente, al menos con los medios de que se dispone hoy día). En un diagrama granulométrico como el de la figura 1 existirán, por lo tanto, dos zonas, indicadas "no apto": si la curva granulométrica del material pasa por una de ellas, aunque sea tan sólo parcialmente, dicho material no es tal que su mezcla con cemento proporcione resultados satisfactorios. En la zona de la izquierde, ello será debido a que contiene demasiada fracción gruesa; en la zona de la derecha, a que contiene demasiada fracción cohesiva (esto es, finos arcillosos).

Otra distinción importante es la que se puede establecer entre los "áridos" (que darán origen, a partir de clerta dosiflcación, a un hormigón magro) y los "suelos" (que darán origen, en análogas condiciones, al suelo-cemento). Claro está que los primeros son más gruesos que los segundos, pero aqui no es tan sencillo diferenciar, puesto que interviene la proporción relativa de las fracciones gruesas y finas. Por lo tanto, se ha recurrido al original criterio de la "línea frontera". La que nos ocupa se designa en la flgura 1 por la letra $A$. En principio, toda curva que pase por debajo de ella dará origen a un hormigón magro, y la que pase por encima dará origen a un suelocemento.

Pero la principal ventaja de la "línea frontera" es que permite distinguir los casos intermedlos, en los que la curva granulométrica del material corta a la línea $A$, dando origen a un producto intermedio entre el hormigón magro y el suelo-cemento propiamente dichos. La distinción puede 
TAMIGES SERIE A.S.T.M.

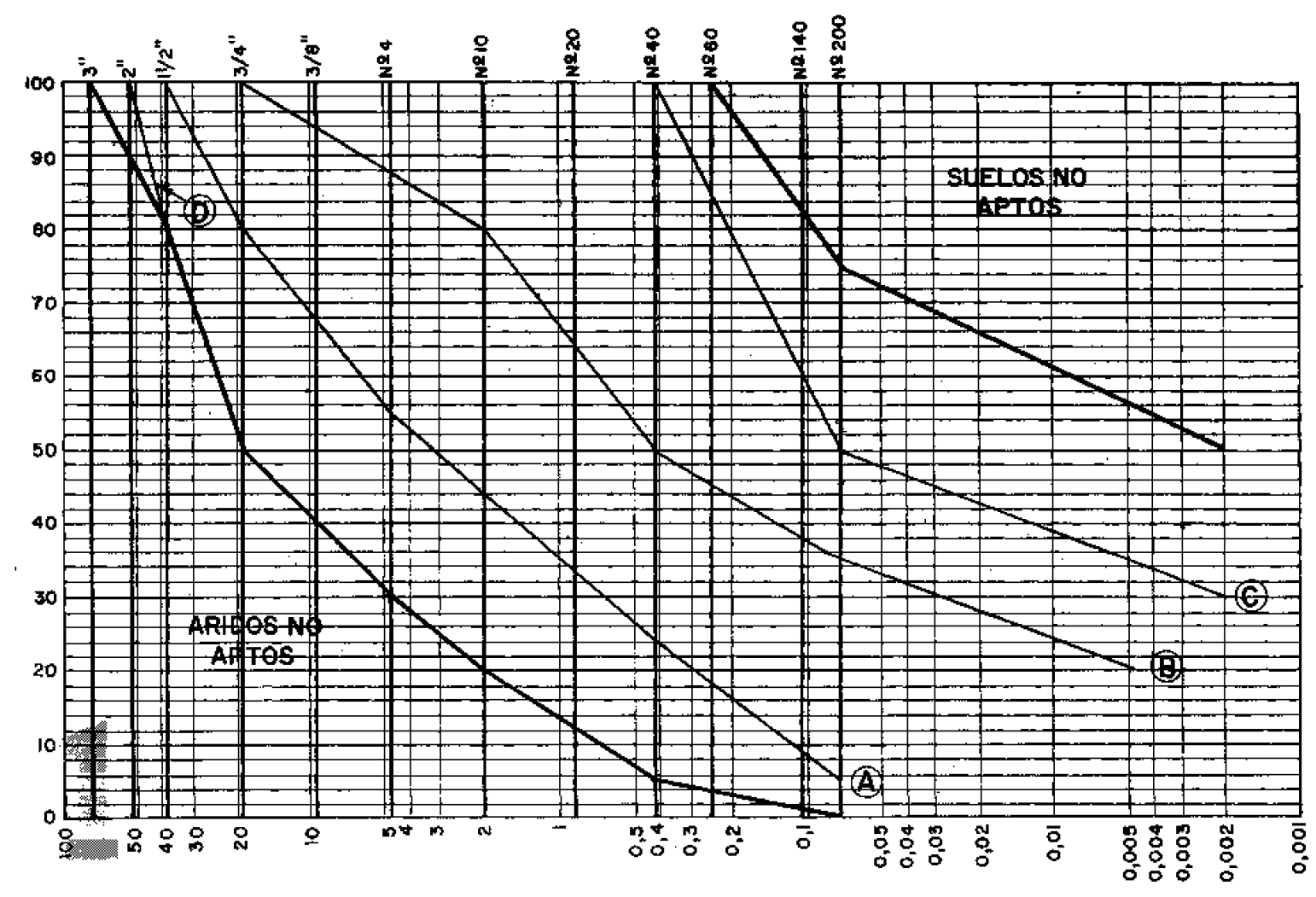

TAMAÑ̃ DE LAS PARTICULAS EN MM.

TAMICES SERIE A.S.T.M.

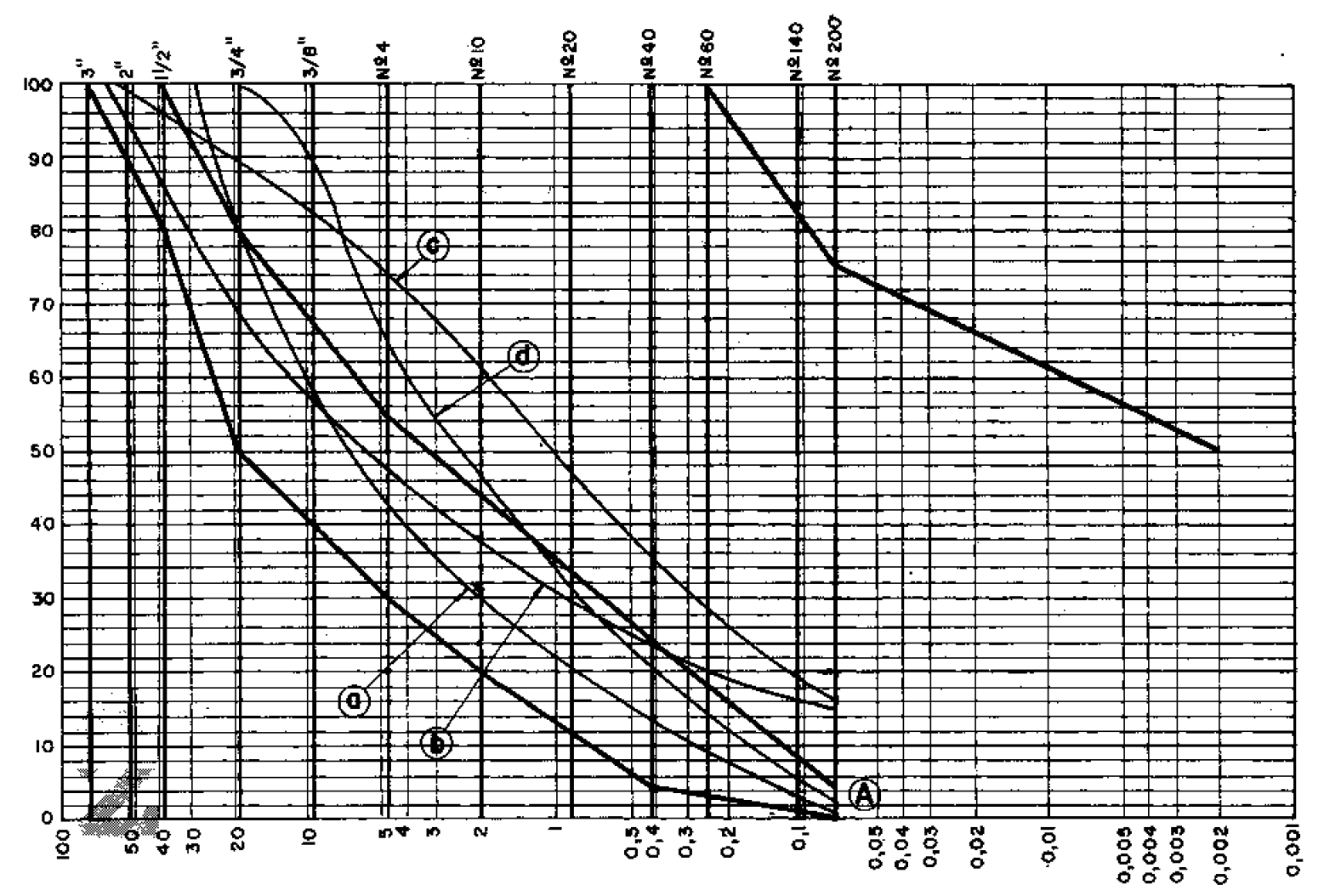

TAMAÑO DE LAS PARTICULAS EN MM. 
TAMIGES SERIE A. S.T.M.

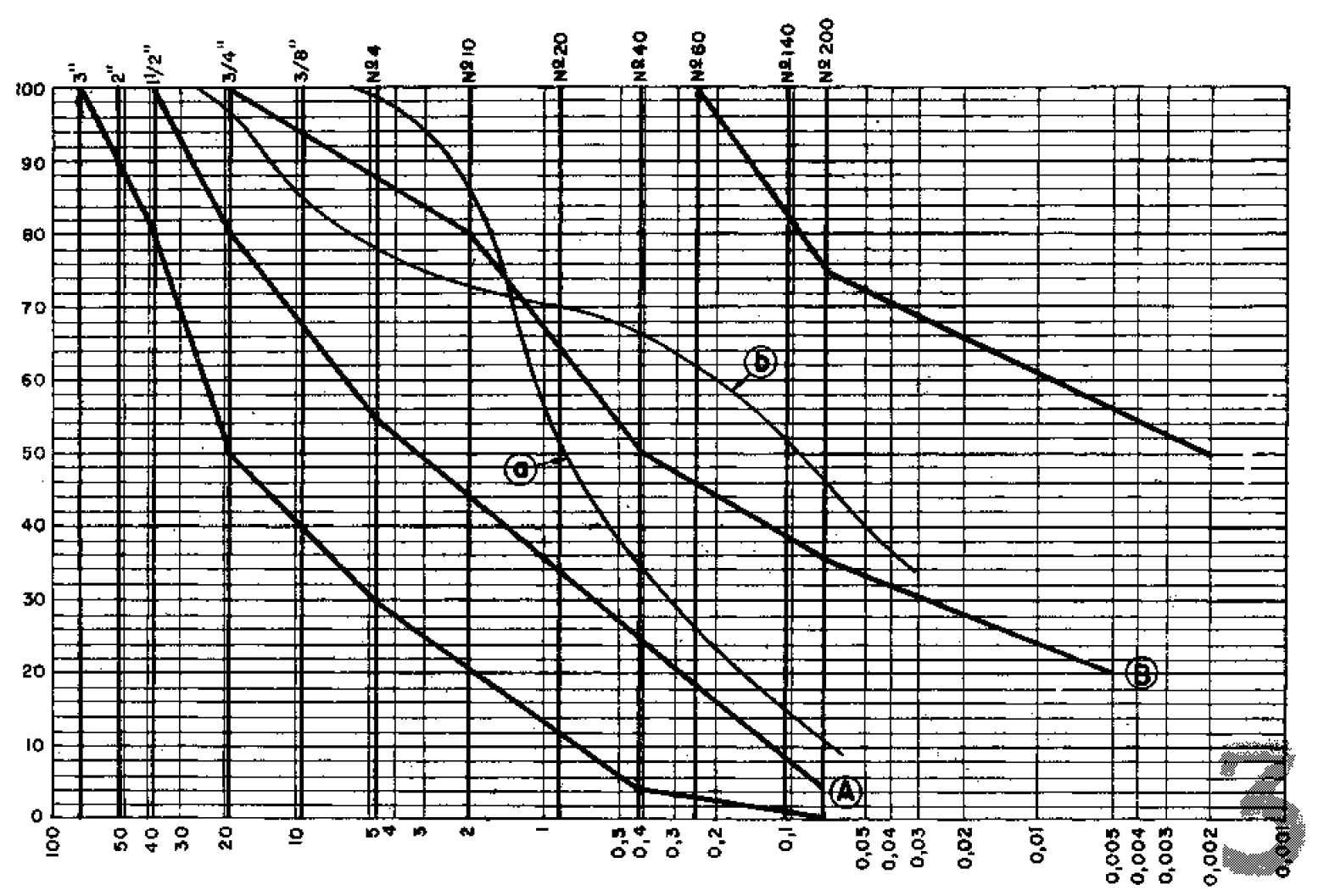

tamaño de LAS PARTICULAS EN MM.

efectuarse, bien sea observando el sentido del corte, bien sea apelando a los coeficientes de uniformidad $\left(d_{00} / d_{10}\right)$ y de curvatura $\left(d^{2}{ }^{3 n} / d_{m n} . d_{1 n}\right)$. De esta manera, se tienen los cuatro casos representados en la flgura 2 :

- Materiales de granulometria uniforme: $\mathrm{C}_{\mathrm{u}}<50$.

Caso a) $\mathrm{C}_{\mathrm{r}}<0,6$ : árido con tamaño máximo reducido (no debe ser inferior a $20 \mathrm{~mm}\left(3 / 4^{\prime \prime}\right)$,

Caso d) $\mathrm{C}_{\mathrm{s}}>0,6$ : suelo con falta de elementos finos (filler), que hace que el conjunto tenga muchos buecos y, por lo tanto, requiera una dosiffcación de cemento más elevada.

- Materiales de granuIometria discontinua: $\mathrm{C}_{\mathrm{n}}>\mathbf{5 0}$.

Caso b) $\mathrm{C}_{\mathrm{c}}<0,6$ : árido con exceso de material flno.

Caso c) $\mathbf{C}_{4}>\mathbf{0 , 6}$ : suelo con exceso de material grueso.

Una vez establecida, como antecede, la casuística completa hormigón magro vs. suelo-cemento, podemos distinguir otros tipos de material, con vistas a propiedades interesantes desde el punto de vista de proyecto (dosificación) y de construcción; también hemos recurrido al empleo de "lineas frontera". Entre ellas destacan (fig. 1):

- La línea B: que divide los suelos "medios" (por debajo) de los suelos "fimos" (por encima): generalmente, los primeros requieren menor dosificaclón de cemento. También aquí se pueden tener casos en los que la curva granulométrica del suelo corta a la línea frontera: materiales de granulometria uniforme [caso a), fg. 3] y discontinua [caso b), flg. 3], los cuales requieren dosiflcaciones más elevadas de cemento.

- La linea $C:$ que se refiere a suelos más finos que los anteriores, cuyo empleo puede ser diflcultado en obra, puesto que se hallan formando terrones cohesivos de difícil disgregación. Se debe exlgir el empleo de maquínaria adecuada. 


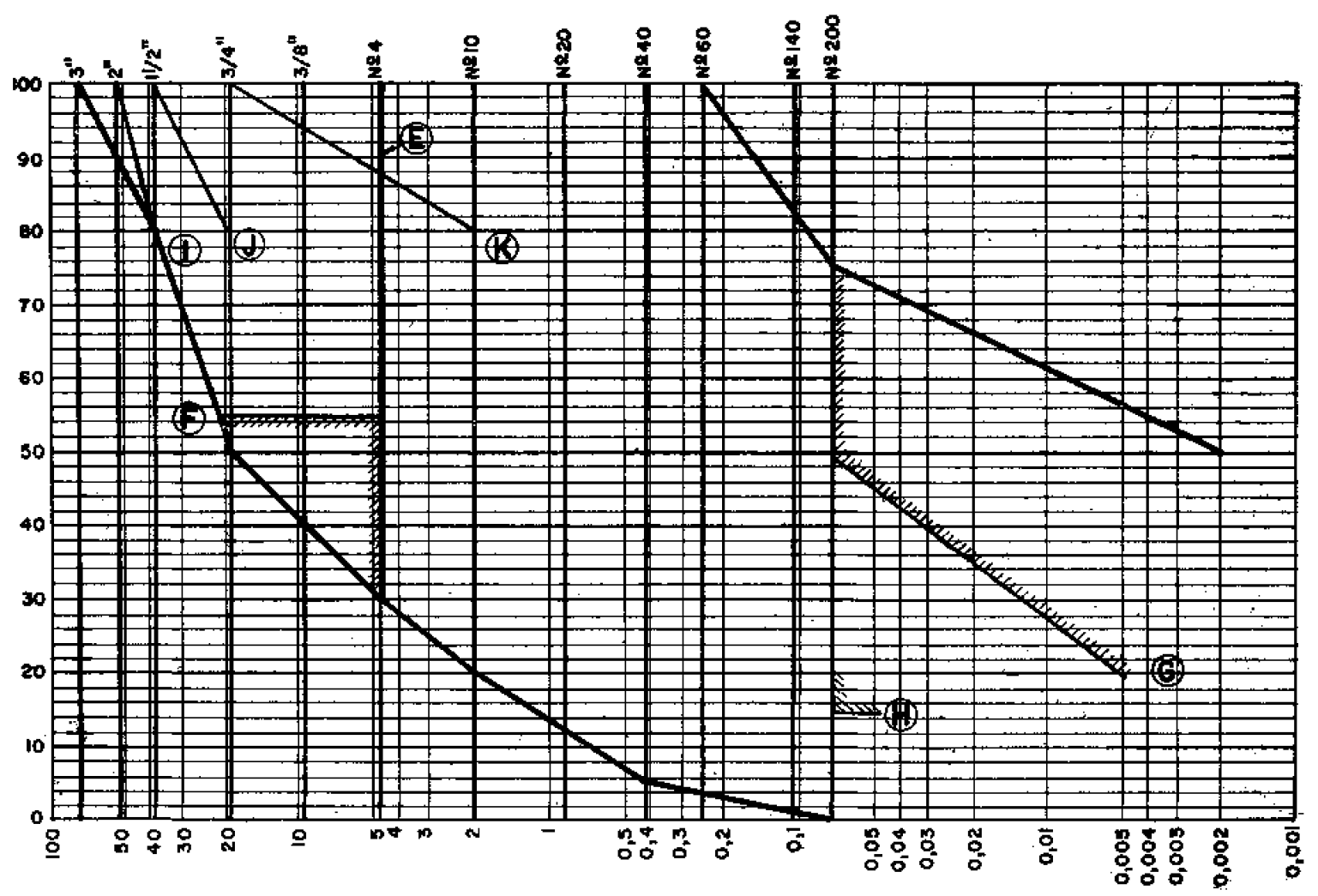

TAMAÑO DE LAS PARTICULAS EN MMi

—La línea D: que se reflere a áridos muy gruesos, cuyo empleo puede ser perjudicial a la maquinaria.

- Las Iíneas de "criterlos de ensayo", marcadas en la figura 4, y que delimitan los campos de aplicación de diversos criterios de dosifleación para suelo-cemento.

- Línea E: Criterios norteamericanos ASTM sobre ensayos de durablidad D-559 (ciclos de inmersión-desecación) y D-560 (ciclos de congelación-deshielo), separa los métodos A (sin gravilla) y $B$ (con gravilla).

-Líneas F y G: Ensayo abreviado de la Portland Cement Association. Se distinguen, asimismo, un método A y $B$, separados por la línea $\mathrm{E}$.

- Linea H: Ensayo del factor de absorción de calcio para detectar suelos que, debico a impurezas generalmente orgánicas, responden anormalmente al anterior ensayo abreviado.

- Líneas I, J, K: Criterios de elección de diámetros de probetas para aplicar las BS-1924, eríterio británico basado en la resistencia a compresión simple.

De esta manera hemos visto cómo aplicando una clasificación cualitativa, basada en el empleo de la línea frontera, se puede predecir, de forma aproximada, no sólo las características más salientes del material en cuanto a su aptitud para la mezcla con cemento, sino también los campos de validez de los diversos criterios de dosiffcación. La combinación de esta clasiflcación cualitativa con un ensayo granulométrico aproximado, puede hacer que los reconocimientos en el campo cobren una mayor envergadura, permitiendo llegar a más conclusiones acerca de la conventencia de emplear determinados materiales.

No basta, sin embargo, el examen de la granulometría y su encaje dentro de uno u otro tipo de material, de los anteriormente definidos; intervienen otras variables, que no hemos considerado aqui, pero que pueden tener una influencia decisiva: principalmente, la plasticidad de la fracción fna, y el contenido en materias orgánicss que inhiben la acción del cemento. De todas estos temas se tratará en sucesivos articulos. 